\title{
Identification of natural plasma emissions observed close to the plasmapause by the Cluster-Whisper relaxation sounder
}

\author{
P. Canu ${ }^{1}$, P. M. E. Décréau ${ }^{2}$, J. G. Trotignon ${ }^{2}$, J. L. Rauch ${ }^{3}$, H. C. Seran ${ }^{3}$, P. Fergeau ${ }^{3}$, M. Lévêque , Ph. Martin $^{3}$, \\ F. X. Sené , E. Le Guirriec $^{3}$, H. Alleyne ${ }^{4}$, and K. Yearby ${ }^{4}$ \\ ${ }^{1}$ CETP/CNRS/UVSQ, 10-12 Avenue de l'Europe,78140 Vélizy, France \\ ${ }^{2}$ LPCE/CNRS and Université d'Orléans, 3A Avenue de la Recherche Scientifique, 45071 Orléans Cedex 2, France \\ ${ }^{3}$ LPCE/CNRS, 3A Avenue de la Recherche Scientifique, 45071 Orléans Cedex 2, France \\ ${ }^{4}$ University of Sheffield, Sheffield, S1 4DU, UK
}

Received: 13 April 2001 - Revised: 17 September 2001 - Accepted: 17 September 2001

\begin{abstract}
We use the data collected by the Whisper instrument onboard the Cluster spacecraft for a first test of its capabilities in the identification of the natural plasma waves observed in the Earth' s magnetosphere. The main signatures observed at the plasma frequency, upper hybrid frequency, and electron Bernstein modes were often difficult to be reliably recognized on previous missions. We use here the characteristic frequencies provided by the resonances triggered by the relaxation sounder of Whisper to identify with good confidence the various signatures detected in the complex wave spectra collected close to the plasmapause. Coupled with the good sensitivity, frequency and time resolution of Whisper, the resonances detected by the sounder allow one to precisely spot these natural emissions. This first analysis seems to confirm the interpretation of Geos observations: the natural emissions observed in Bernstein modes above the plasma frequency, now widely observed onboard Cluster, are not modeled by a single Maxwellian electrons distribution function. Therefore, multi-temperature electron distribution functions should be considered.
\end{abstract}

Key words. Space plasma physics (active perturbation experiments; waves and instabilities; instrument and techniques)

\section{Introduction}

Plasma waves observed by spacecraft in planetary magnetospheres and beyond are good tracers which allow for a diagnosis of the local or remote particle populations from which they originate. They can thus provide key information on the physical mechanisms taking place in the plasma. However, the understanding of the source mechanism requires first a precise and reliable identification of the propagating modes of the observed emissions. This can be difficult given the wide variety of characteristic frequencies of the existing plasma waves. For example, the electron plasma fre-

Correspondence to: P. Canu (Patrick.Canu@ cetp.ipsl.fr) quency, $F p$, and the characteristic frequencies of the Bernstein modes (the upper hybrid frequency, $F u h$, the harmonics of the electron gyrofrequency, $n F c$, and the so-called $F q$, which is the maximum frequency for each mode between two consecutive gyroharmonics and corresponds to a zero group velocity, as well as the electromagnetic ordinary, $F o$, the extraordinary $F x$ and $F z$, and the cutoff frequency of the $Z$ mode radiation $\left(F z=-F c / 2+\left(F c^{2} / 4+F p^{2}\right)^{1 / 2}\right)$ are all situated in the same frequency range and have to be taken into account when trying to identify the plasma emissions detected by a spacecraft.

By stimulating some of the main characteristic frequencies of the surrounding plasma, the relaxation sounder offers a key reference frame from which the natural emissions can be identified. The capabilities of this instrument were soon illustrated after its first operations on the Geos spacecraft (Christiansen et al., 1978a and 1978b). Further examples of the capabilities of this technique were provided inside the Earth's magnetosphere and solar wind by similar instruments flown on board Geos-2 and Isee-1 (Etcheto et al., 1983; Belmont et al., 1984), Viking (Perraut et al., 1990), and in the Jovian magnetosphere by Ulysses (Le Sager et al., 1998; Moncuquet et al., 1997). However, despite the advances provided by these instruments, key questions were still not fully resolved. For example, the simultaneous and unambiguous observations of both the plasma frequency, $F p$, and the upper hybrid frequency, $F u h$, evident in ionospheric topside sounder data (McAfee, 1974) due to their canonical relationship, $F u h^{2}=F p^{2}+F c^{2}$, were only reported on the Viking satellite (Décréau et al., 1987) and not from high altitude sounders such as the Geos sounder (Etcheto and Bloch, 1978). $F p$ and $F u h$ were alternatively used to name the first resonance that was not a gyroharmonic, without clear justification (see Christiansen et al., 1978b). Consequently, the same terminology was used to identify the strongest plasma wave which closely matched this resonance, without a clear identification of $F p$ or $F u h$. Furthermore, the data suggested that the peak emissions could be located sometime at the first Fq (Christiansen et al, 1978a, 1978b; Etcheto et al., 1983). 
The situation was further complicated when it was shown that the observed resonances were not dependent on a single Maxwellian electron distribution function (Belmont, 1981; Etcheto et al., 1983). Since all of these key resonances have a frequency separation less than one gyroharmonic, which in this case is small compared to $F p$, the induced uncertainty in the diagnosis of the electron density was almost negligible. However, this lack of precision can be a bigger problem for wave mode identification. Due to the difficulty of the reliable identification of some of these emissions, some interpretation of the origin of commonly observed wave signatures, such as the continuum radiation, lead to controversial interpretation (Etcheto et al.,1985).

The Whisper relaxation sounders on board each of the Cluster spacecraft have been build on the basis of the Geos and Isee sounders, with their characteristics adapted to the requirements of the Cluster objectives in terms of frequency range and time resolution (Décréau et al., 1997). The purpose of this paper is to test and to illustrate the capabilities of the instrument in the identification of natural plasma waves through some examples collected during the commissioning period. A more general report on the first Whisper observations can be found in a companion paper (Décréau et al., 2001, this issue). Time resolution and instrument sensitivity are an order of magnitude better in routine operations than on these previous spacecraft. Better time and frequency resolution are available during the operations of the WideBand instrument, but are limited in time (Gurnett et al., 2001, this issue). The frequency resolution of the resonance observed by Whisper $(160 \mathrm{~Hz})$, although better than the standard one used both for passive and active measurements on Geos $(300 \mathrm{~Hz})$ and Isee $(400 \mathrm{~Hz})$, is not reaching the best one available on the Geos sounder in the active mode $(12 \mathrm{~Hz})$. But the identification of a plasma wave can be better performed with Whisper due to its higher sensitivity (an order of magnitude) which can track these emissions at a lower level and its better time resolution. Since a diagnosis of an active spectrum requires that the characteristic frequencies $F c e$ and $F p$ be stationary during the whole frequency sweep of the instrument, the identification of natural emissions, sometimes very unstable signatures (Gough et al., 1981b), requires that the plasma parameters be stable for more than $20 \mathrm{~s}$ with these past instruments, whereas now it is only about $2 \mathrm{~s}$ with Whisper in standard mode. Moreover, simultaneous observations from close spacecraft can be very enlightening, as it helps to point out stationary signals.

For this very preliminary study, we have considered spectra observed just outside the plasmapause due to their richness and complexity. The $F p / F c$ ratio, which is observed at about 3 , allows for the instrument to clearly discriminate between $F p$ and $F u h$. The second part of the paper briefly describes the instrument. The third part presents the observations with their interpretation found in the fourth part, and the last part summarizes what can be expected during the Cluster mission.

\section{The instrument}

The Waves of High frequency and Sounder for Probing the Electron density by Relaxation (WHISPER) experiment is a part of the Wave Experiment Consortium (WEC, Pedersen et al., 1997) flying on board each of the Cluster spacecraft. The objectives of Whisper are two fold: To measure the total electron density via active stimulation of the surrounding plasma in the so-called active modes, and to provide a survey of the natural emissions in the frequency range $2-80 \mathrm{kHz}$ during the passive modes. The detection of the electric signal relies on sphere antennae located on symmetric radial booms of 88 meters in length, tip to tip, which are part of the Electric Field and Wave experiment (Gustafsson et al., 1997; Gustafsson et al., 2001, this issue). The main difference between Whisper and the past relaxation sounders flown on Geos, Isee1 and Viking is that instead of analyzing the electric signal by sweeping the frequency range with a Stepped Frequency Analyzer (SFA), the instrument relies heavily on onboard data processing. The electric waveform is collected over the Whisper frequency range and the frequency spectrum is obtained through onboard Fast Fourier Transform every $13 \mathrm{~ms}$. On active modes, a pulse with a duration of $1 \mathrm{~ms}$ or $0.5 \mathrm{~ms}$ excites a 1 or $2 \mathrm{kHz}$ frequency bandwidth that is sent over the whole frequency range in 40 or 80 steps. The frequency resolution can be adapted by selecting the bin numbers of the FFT, which can be either $64,128,256,512$, corresponding to a frequency resolution of $1.2 \mathrm{kHz}, 600 \mathrm{~Hz}, 320 \mathrm{~Hz}$ and $160 \mathrm{~Hz}$; this lower and last value is always used in sounder modes. A complex data selection process is then performed on the signal by the Digital Wave-Processing experiment (DWP) in order to adapt the data flow to the allowed telemetry rate (see Woolliscroft et al., 1997). The natural emissions detected in the passive mode are averaged. The bins corresponding to the frequency range stimulated by the emitter are selected, and depending on the mode of operations, additional information such as the active/passive amplitude ratio or passive information collected during the active mode can be transmitted on the ground. The time resolution allowed by the onboard data processing within the allocated bit rate of about $1 \mathrm{kbit} / \mathrm{s}$ in normal mode is typically about $1.5 \mathrm{~s}$ for the active mode and $2 \mathrm{~s}$ for the passive measurements. The duty cycle choosen within WEC allows are to collect two active spectra every $28 \mathrm{~s}$ in normal mode (see Pedersen et al., 1997). The instrument's overall sensitivity (averaged noise level) is about $210^{-7} \mathrm{~V} / \mathrm{Hz}-{ }^{1 / 2}$. For further details on Whisper, see Décréau et al. (1997) and Décréau et al. (2001, this issue).

The four spacecraft of the Cluster mission have been launched in pairs on 16 July 2000 and 12 August 2000, respectively. The beginning of the commissioning phase was marked by reaching the constellation orbit with a perigee of $19000 \mathrm{~km}$, and an apogee of $120000 \mathrm{~km}$ with an inclination of $90^{\circ}$. The commissioning phase extended through December 2000. Although primarily devoted to the test of the instruments and their various operating modes, a wealth of interesting observations have been collected. A first example of 


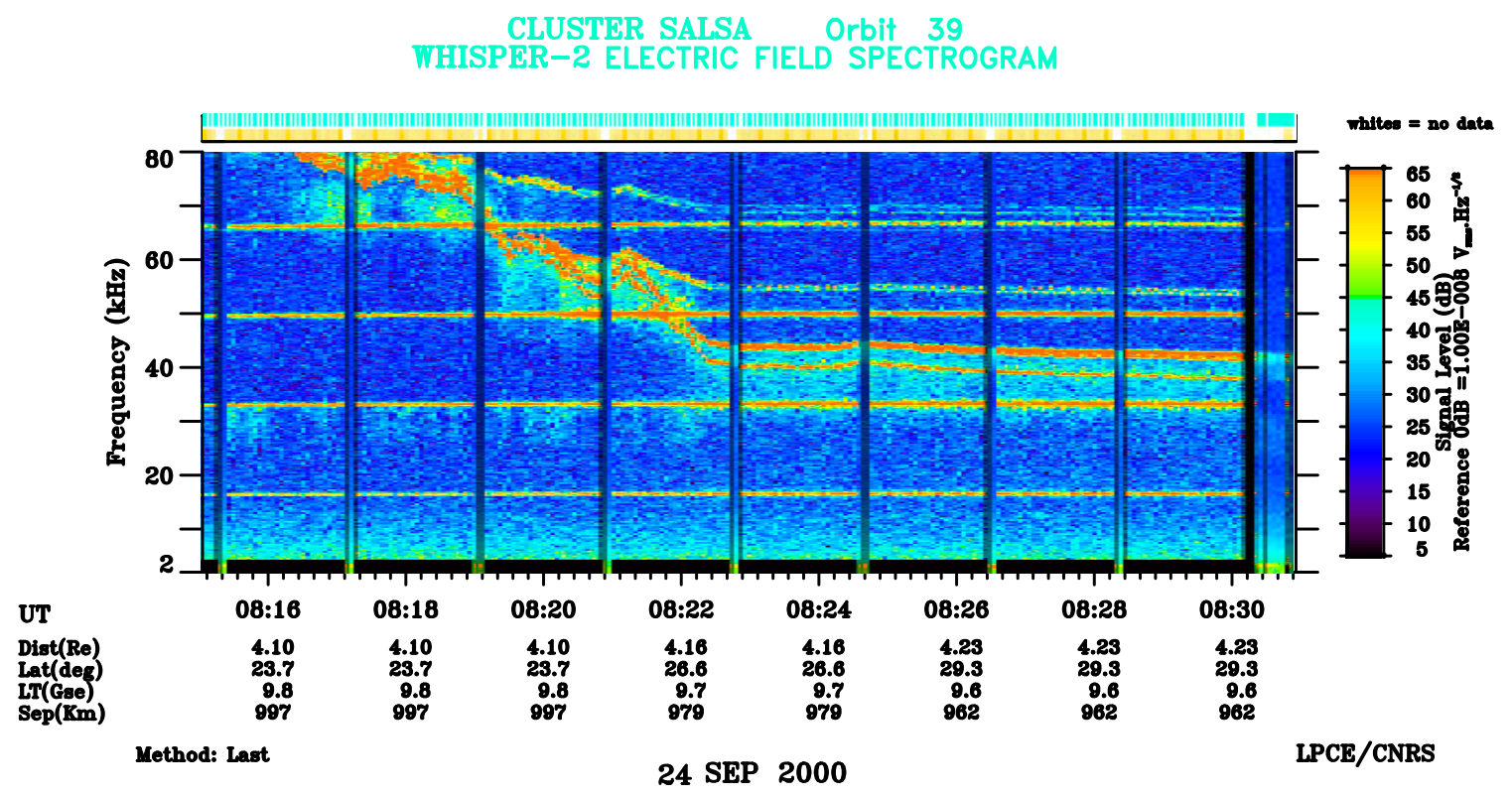

Fig. 1. Dynamic spectrogram of the electric field spectral densities observed by Whisper on 24 September 2000 from Cluster 2 Salsa. The instrument was in active mode for most of the period displayed. The strong horizontal lines are resonances at the harmonic of the local electron gyrofrequency. Resonances at $F p, F u h$ and $F q$ seen at decreasing frequencies until 08:22 UT are evident between the harmonics.

combined observations of resonances stimulated by the emitter of Whisper and natural plasma emissions is presented in Décréau et al. (2001, this issue). We are showing here in further detail some examples in order to illustrate, on one hand, the variety and sometimes the complexity of the wave spectra, and on the other hand, the instrument's capabilities and eventually its limits in the detection and the identification which can be achieved by Whisper of the natural plasma waves.

\section{Observations}

We begin first with the simplest and almost textbook-like, illustration of the observation of active resonances in frequency spectra. Figure 1 presents a dynamic spectrogram of Whisper data, with the colour-coded amplitude displayed as frequency versus time, collected by the Cluster 2 Salsa spacecraft on 24 September 2000, as the satellite was exiting the dense plasmasphere. The spacecraft geocentric distance was $4.15 R_{E}$, the latitude and local time in the GSE system were $23^{\circ}$ and 9.8 at the beginning of the sequence. The instrument was run in active mode for most of the time, except for two natural spectra which were sampled consecutively about once every $2 \mathrm{~min}$. They are identified on the plot by the vertical black bars. Throughout the paper, the frequencies mentioned for characterizing the emissions will be those of the Whisper bin frequency where the peak amplitude is observed. The strong (red) horizontal lines at almost constant frequencies of $16.64,33.37,49.97$ and $66.57 \mathrm{kHz}$ at 08:22:30 UT, for example, are the local electron gyrofrequency and its harmonic, $n F c$, with $n=2,3,4$. The addi- tional resonances which enter the frequency range of Whisper at $80 \mathrm{kHz}$ at 08:17 UT are dependent on the local electron density, which is decreasing rapidly as the spacecraft recedes from the Earth. We can identify three main resonances at the beginning of the sequence, close to $08: 18 \mathrm{UT}$, between $4 n F c$ and $5 n F c$ (out of scale).

As the frequency of the two lowest frequencies decrease, the third one drops asymptotically down toward the $4 n F c$ gyroharmonic. As the two main resonances cross the $4 n F c$ harmonic, a third resonance appears in the same branch, but located above them and again, its frequency decreases asymptotically toward the bottom harmonic of the branch. In the final part of the plot, between 08:23 and 08:30 UT, the various resonances in each branch can be observed at almost a constant frequency, indicating a constant electron density. The behaviour of the non-harmonic resonances with respect to the $n F c$ is precisely what one can expect from waves propagating at the zero-group velocity of the Bernstein modes, when the $F p / F c$ ratio decreases from about 4 down to about 2.5 (Etcheto and Bloch, 1978). The first two resonances, which are not $n F c$, have frequencies of, respectively, $41.18 \mathrm{kHz}$ and $44.43 \mathrm{kHz}$ at $08: 24: 35 \mathrm{UT}$. Their frequency separation matches $F u h^{2}=F p^{2}+F c^{2}$ with $F c=16.68 \mathrm{kHz}$. The resonances in the upper branch represent the so-called $F q$, which are the highest frequencies of the mode in each branch and correspond to the zero-group velocity solutions. The $F q$ above $4 n F c$ seems to be split, which is a known feature already reported on Geos (Higel, 1978), and regularly observed on Whisper. A question can arise at this point. If the three resonances expected from the Bernstein modes, $F p, F u h$ and $F q$, are indeed observed before 08:21 UT, no $F q$ is seen above $F u h$ in the same gyro- 

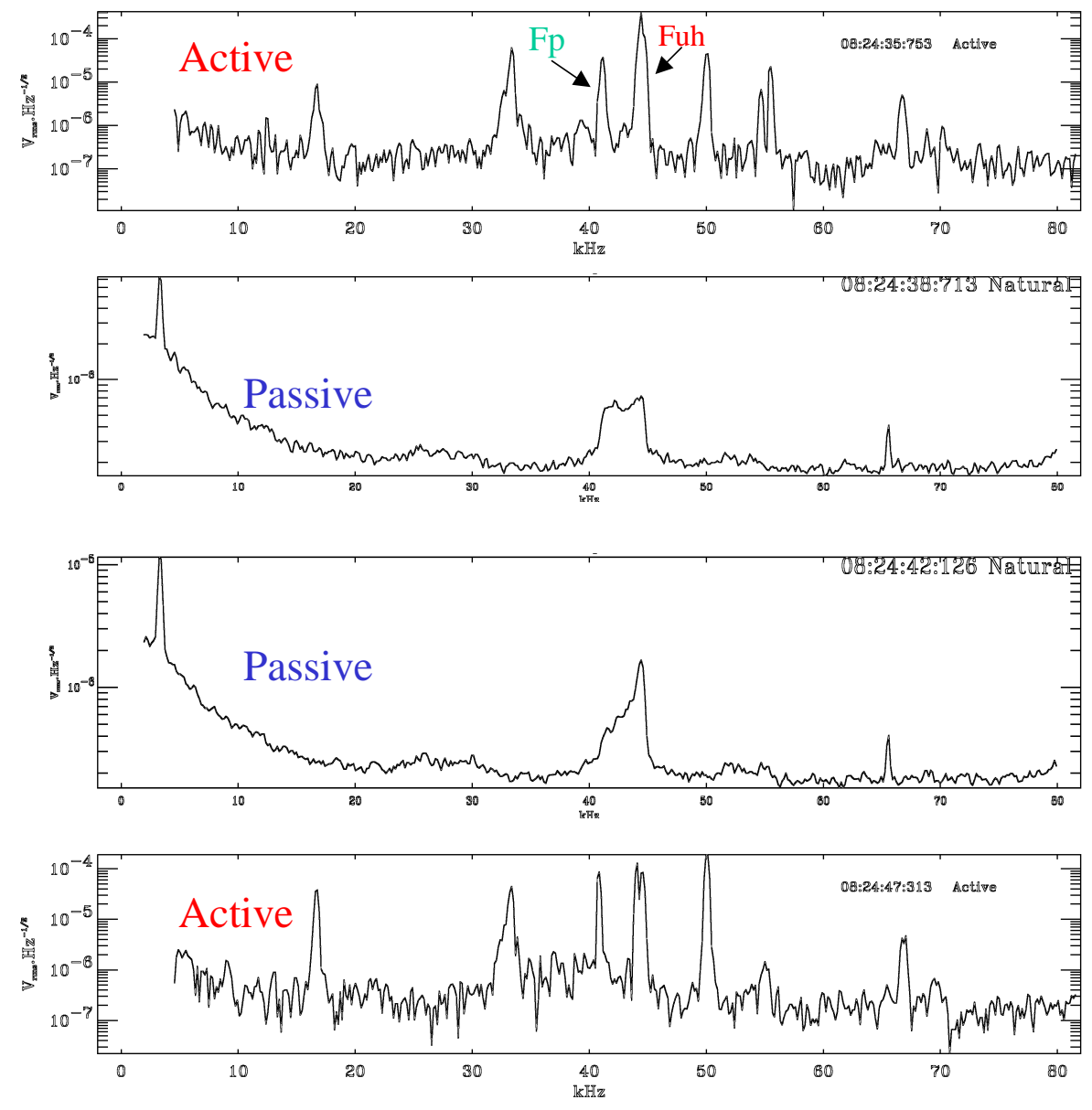

Fig. 2. Consecutive active and passive amplitude versus frequency spectra. Time separation between spectra is $2-3 \mathrm{~s}$. The enhanced natural plasma emission has its low and high cutoff at $F p$ and $F u h$, respectively. The emission at $F u h$ is seen as unstable on the second passive spectrum. harmonic branch after that time when $2 F c<F u h<3 F c$. This could be due to the fact that $F u h$ and $F q$ are very close in frequency, which can happen for some given $F p / F c$ ratios (Crawford, 1964). It is then difficult to clearly separate them with the frequency resolution of Whisper $(160 \mathrm{~Hz})$. At this stage, we will not try to discriminate further, since a better frequency separation will be provided in the next example. A complete diagnosis of the resonances observed in Figure 1 is provided in a companion paper (Trotignon et al., 2001, this issue). Figure 2 shows four consecutive spectra, with amplitude versus frequency, obtained during this period, each separated from the previous one by about $3 \mathrm{~s}$. The top one is an active spectrum and it displays the resonances identified in Fig. 1, with $F u h$ representing the strongest one, with an amplitude, $A \sim 4 \cdot 10^{-4} \mathrm{~V} / \mathrm{Hz}^{1 / 2}$, about two orders of magnitude above the background noise. The bottom active spectrum when compared to the top spectrum highlights some time variations of the resonance amplitude in the $12 \mathrm{~s}$ that separate the active periods. It suggests, moreover, that indeed there are two resonances close to $F u h$, with the upper one tentatively identified as the expected $F q$ discussed above. The two middle spectra are collected in passive mode. The mode used here corresponds to the best frequency resolution for passive spectra $(160 \mathrm{~Hz})$, and each spectrum is an onboard average of 64 spectra, even $13 \mathrm{~ms}$ in duration. A large bandwidth signal is observed, with an amplitude of $A \sim 6 \cdot 10^{-7} \mathrm{~V} / \mathrm{Hz}^{1 / 2}$ between 41 and $43 \mathrm{kHz}$. The lower frequency cutoff matches exactly the $F p$, while the upper cutoff corresponds exactly to $F u h-F q$. On the second passive spectrum, the signal increases by a factor of two over a narrow bandwidth, with a frequency matching the $F u h-F q$ seen in the active mode. Rapid increases in the amplitude of $F u h$ were observed on Geos (Gough et al., 1981b). However, the limited sensitivity of the instrument that allows one to observe only emissions with an amplitude above $\sim 10^{-6} \mathrm{~V} / \mathrm{Hz}^{1 / 2}$ would have hidden, in this case, all passive features in the $F p-F u h$ band, except the emission excited at $F u h$ on the second spectrum.

The natural emissions observed by Whisper on 25 November 2000 are far more complex than those presented in Fig. 1. Figure 3 shows a dynamic spectrogram of the Whisper data obtained that day on Cluster 1 Rumba, while the spacecraft was again exiting the plasmasphere. The distance was increasing from 3.94 to $4.5 R_{E}$, the latitude was increasing from $12.3^{\circ}$ to $40^{\circ}$, and the local time was decreasing from 6.1 to $5.2 \mathrm{~h}$ during the time interval considered here. The plasmapause and its vicinity is one of the regions known to be at the origin of the electromagnetic continuum radiation (Gurnett, 1975; Jones, 1982; Etcheto et al., 1982; and Barbosa, 1982), as well as the source of strong electrostatic, 


\section{CLUSTER RUMBA Orbi† 65 WHISPER- 1 ELECTRIC FIELD SPECTROGRAM}

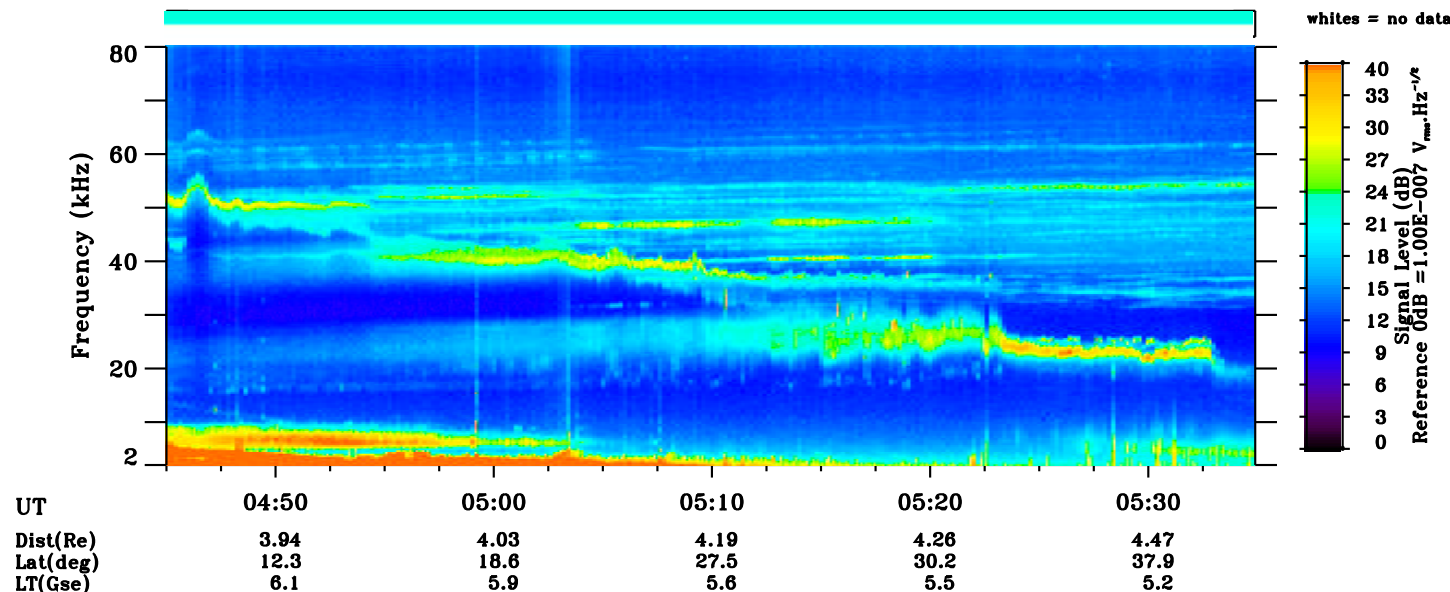

25 NOV 2000

LPCE/CNRS

Fig. 3. Dynamic spectrogram obtained from Whisper data on 25 November 2000 from Cluster 1 Rumba. The instrument was in passive mode only. A mixture of narrow band emissions at constant frequencies and stronger waves with decreasing frequencies are observed as the spacecraft recedes from the plasmasphere.

\section{CLUSTER TANGO Orbi† 65 WHISPER-4. ELECTRIC FIELD SPECTROGRAM}

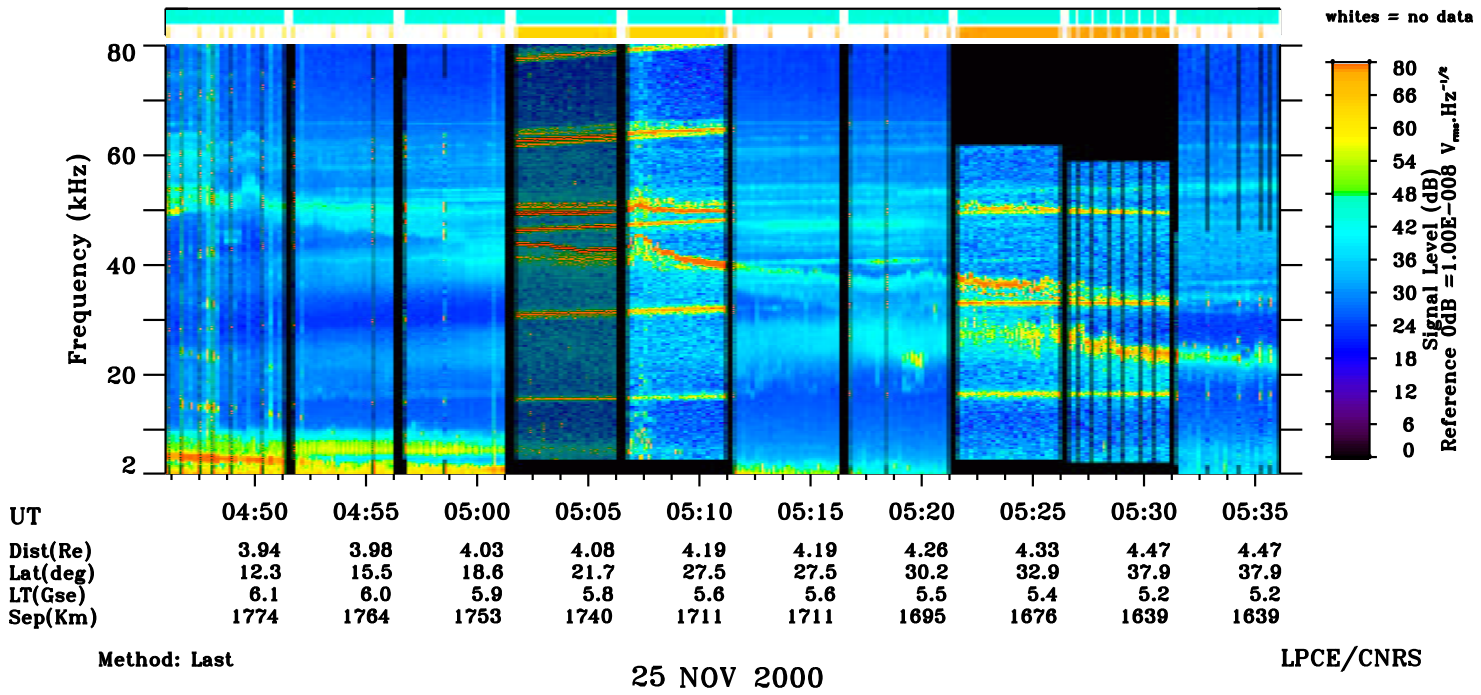

Fig. 4. Same period as in Fig. 3 but obtained from Cluster 4 Tango. Whisper was in active mode part of the time, as indicated by the yellow-orange bar at the top of the spectrogram. The strong resonance is associated with the strong decreasing frequency.

locally observed emissions (Christiansen, 1978b; Gough et al., 1979; Gough et al., 1981a; Canu, 1984). The local gyrofrequency $F c$ is increasing slightly during this period, from about $F c=14.5 \mathrm{kHz}$ at 04:50 UT to $F c=16.4 \mathrm{kHz}$ at 05:30 UT. The strong emissions seen before 05:10 UT at frequencies below $10 \mathrm{kHz}$ are whistler emissions similar to those reported by the WideBand instrument on Cluster (Gurnett et al., 2001, this issue). Two features dominate the Whis- per spectrogram. First, a collection of narrow band emissions, with almost constant frequencies during the period displayed, and second, strong emissions, with a peak decreasing in frequency from about $50 \mathrm{kHz}$ at the beginning of the plot to about $20 \mathrm{kHz}$ at the end. The narrow band emissions are the fine structures within the misnamed continuum radiation, identified first from Isee-1 data (Kurth et al., 1981). The strongest emission does not always have a large amplitude. 

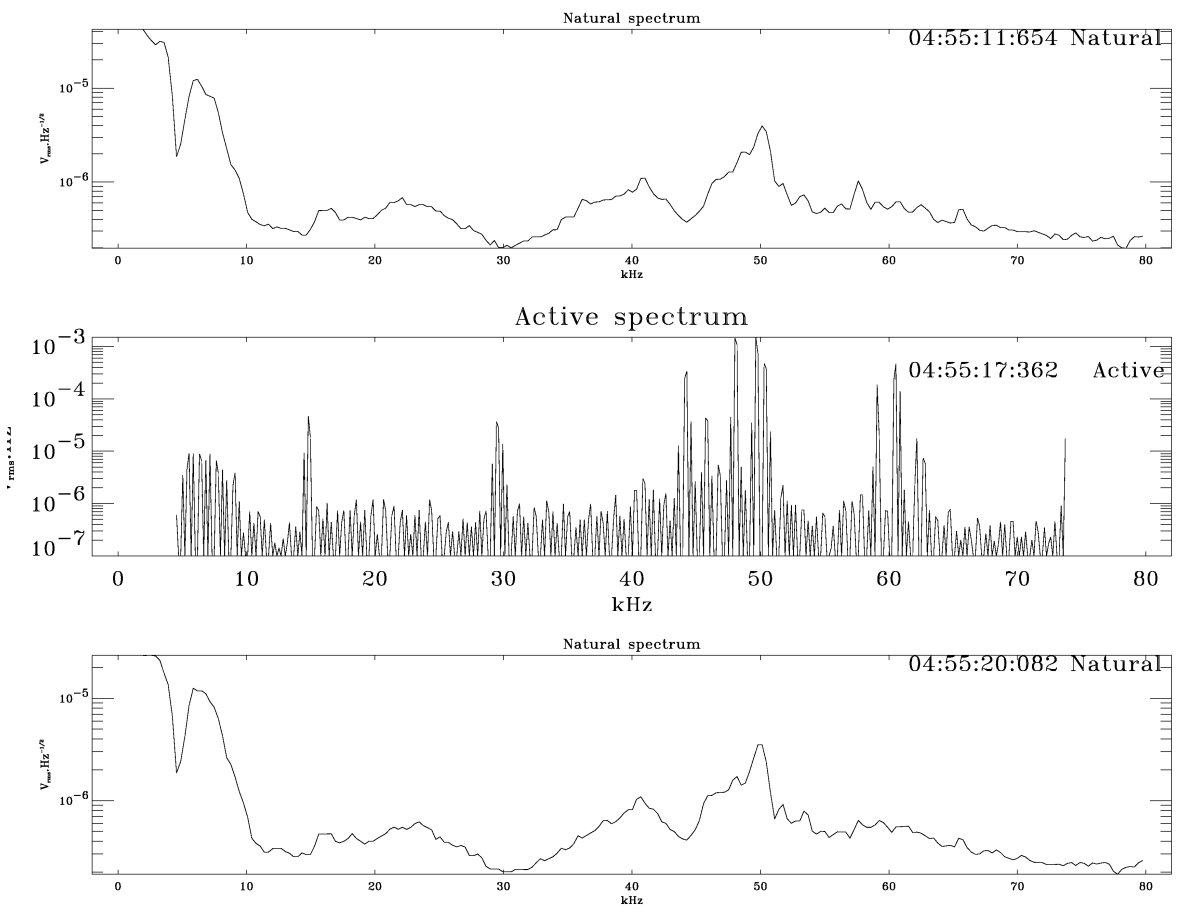

Fig. 5. Consecutive passive and active spectra collected on Tango, a few seconds apart, at 04:55 UT on 25 November. The resonances at $44.27,45.74$, 48.01 and $49.8 \mathrm{kHz}$ are $3 F c, F p, F u h$ and $F q$, respectively. The natural emission band lies between $F p$ and $F q$, with $F q$ representing the strongest wave.
This amplitude seems to fade away when its frequency approaches a gyroharmonic (not shown on the plot), while the amplitude of the emission in the gyroharmonic branch below increases. The maximum of the amplitude seems to occur when the frequency is close to the middle of the harmonic branch. Whisper 1 Rumba was then only collecting passive measurements, so no resonance spectra were available. But the same region was sampled at the same time by Cluster 4 Tango, with Whisper in active mode part of the time on this spacecraft, as shown in Fig. 4. The separation distance between both spacecraft was about $1700 \mathrm{~km}$. The different sequences, separated by some data gaps (vertical black bars), correspond to the various modes tested, since Whisper was still in its commissioning phase on Tango. The active periods are identified by a bar at the top of the plot in yellow, when the transmitter voltage is set to $100 \mathrm{~V}$, and in red colour when it is set to $200 \mathrm{~V}$. One can check that the natural emissions observed here closely match the ones observed by Rumba at the same time. The strongest resonance and the first with increasing frequency seen outside the $n F c$ series corresponds to the strong natural emission, which decreases in frequency during the period. Figure 5 displays consecutive passive and active spectra obtained at about 04:55 UT, which allows for a more precise view of the resonances and an identification of natural emissions. At this time, the bit rate dedicated to the active mode allows one to transmit only half of the data points. The onboard data processing then pairs two adjacent bins and downloads the characteristics of the strongest one (see Décréau et al., 1997). The natural emissions are analyzed on board with the 256 bins FFT which provides a $320 \mathrm{~Hz}$ frequency resolution. On the active spectrum (middle plot), the $n F c$ are well identified at 14.81, 29.46, 44.27 and
$59.08 \mathrm{kHz}$. Fuh is detected at $48.01 \mathrm{kHz}$, while $F p$ is the faint peak observed at 45.74 , between $3 F c$ and $F u h$. The strong resonance at $49.8 \mathrm{kHz}$ above $F u h$ is a $F q$, which is seen in Fig. 4 with a frequency that decreases asymptotically toward $3 F c$ at about 05:10UT. This $F q$ matches the frequency of the strongest emissions observed close to $50 \mathrm{kHz}$ on the natural spectra (top and bottom plots) that are collected a few seconds before and after. A small bump can be observed below this strong emission, with a frequency matching the frequency of the $F u h$ resonance. Multiple low power peaks are clearly observed in Fig. 4 and Fig. 3, above the strong amplitude $F q$. At this stage of the study, it is not possible to precisely identify each of these secondary peaks, which are seen with an almost constant frequency and probably propagating narrow band continuum emissions that are non-locally produced. The possibility that some of the stronger $F q$ emissions at 04:55 UT or 05:15 UT, for example, could contribute to the generation of the continuum radiation also needs to be further investigated. The reader should note, however, that the peaks observed here have a rather low amplitude, about $1 \mu \mathrm{V} / \mathrm{m}$, which is far below the strong emissions, above $1 \mathrm{mV} / \mathrm{m}$ reported by spacecraft such as Geos, Isee, Ampte or Geotail. Such strong emissions are known to be concentrated close to the geomagnetic equator (Christiansen et al., 1978b; Gough et al., 1979; Gough et al., 1981a; Canu, 1984). Their frequency behaviour has been recently statistically studied from Ampte data (Labelle et al., 1999). They are also observed by Whisper, but will not be discussed here.

We can also point out the featureless two bands of enhanced emissions between $F c$ and $2 F c$, and $2 F c$ and $3 F c$. These broad band, diffuse emissions are well-know features 

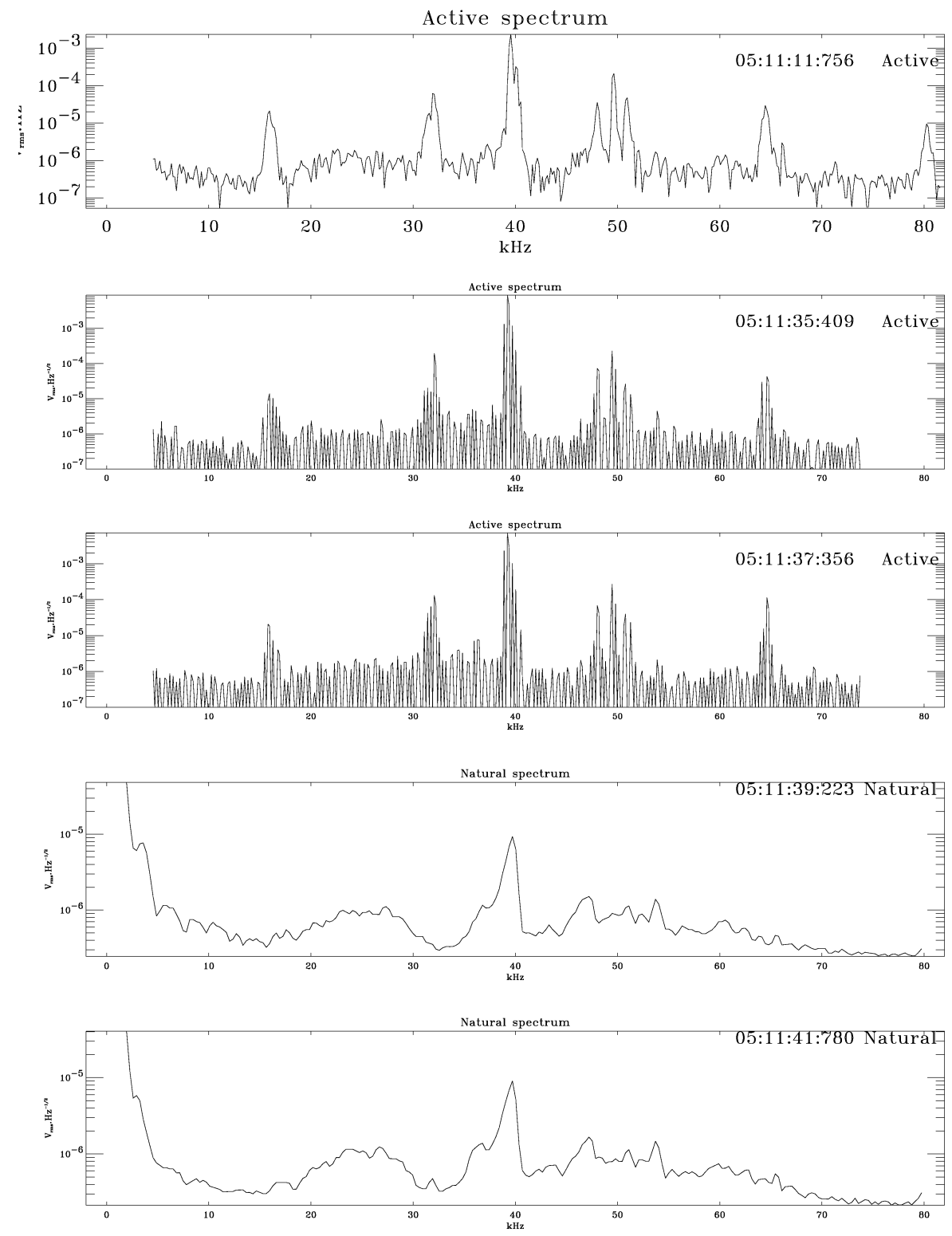

Fig. 6. Consecutive active and passive spectra collected on Tango at 05:11 UT on 25 November. On the active spectra, the resonance at $F p$ is barely visible at $36.4 \mathrm{kHz}$ on the middle spectrum. Fuh and $F q$ are too close in frequency to be clearly separated. of terrestrial magnetospheric wave spectra (Christiansen et al., 1978b). They have also been observed in the Io plasma torus from the Ulysses-Urap data and shown to represent quasi-thermal noise on the Bernstein modes (Meyer-Vernet et al., 1993; Moncuquet et al., 1997). Their amplitude increases while Cluster approaches the region where the strongest emission enters the corresponding band (see Fig. 3 at 05:10UT). Figure 6 shows consecutive active and passive spectra obtained close to 05:11 UT on Tango. Once again, the low frequency cutoff of the natural emissions matches the $F p$ resonance, which this time is barely visible at $36.4 \mathrm{kHz}$ (panel 3 from top). $F u h$ and the first $F q$ are so close that they can barely be separated at about $39.55 \mathrm{kHz}$ (see top panel displaying all frequency lines). More generally, the amplitude of the resonance at $F p$ and the one of the natural emission between $F p$ and $F u h$ are varying with time.
Very often during the period considered here, the resonance at $F p$ cannot be sorted out from the background noise. A first analysis of the data suggests that this variation is related to the spin phase. The strong directivity of the resonances triggered by a relaxation sounder are well-known (Higel, 1978); but we will not investigate this point in this work. Returning to Fig. 4, we can now identify $F p$ as the lower cutoff of the fuzzy emissions seen just below the strongest $F q$ related emissions, clearly at 04:55 UT, and close to $45 \mathrm{kHz}$.

Another example is provided in Fig. 7, which displays a Whisper spectrogram collected from Cluster 1 Rumba on 1 October 2000. Once again, the spacecraft was leaving the plasmasphere, close to the perigee: The geocentric distance was about $4 R_{E}$, with the latitude varying from $7^{\circ}$ to $+19^{\circ}$, and the local time was close to $10 \mathrm{~h}$. The gyrofrequency is about $12.8 \mathrm{kHz}$ at $10: 50 \mathrm{UT}$ and increases slightly dur- 


\section{CLUSTER RUMBA
Orbi† 42
WHISPER-1 ELECTRIC FIELD SPECTROGRAM}

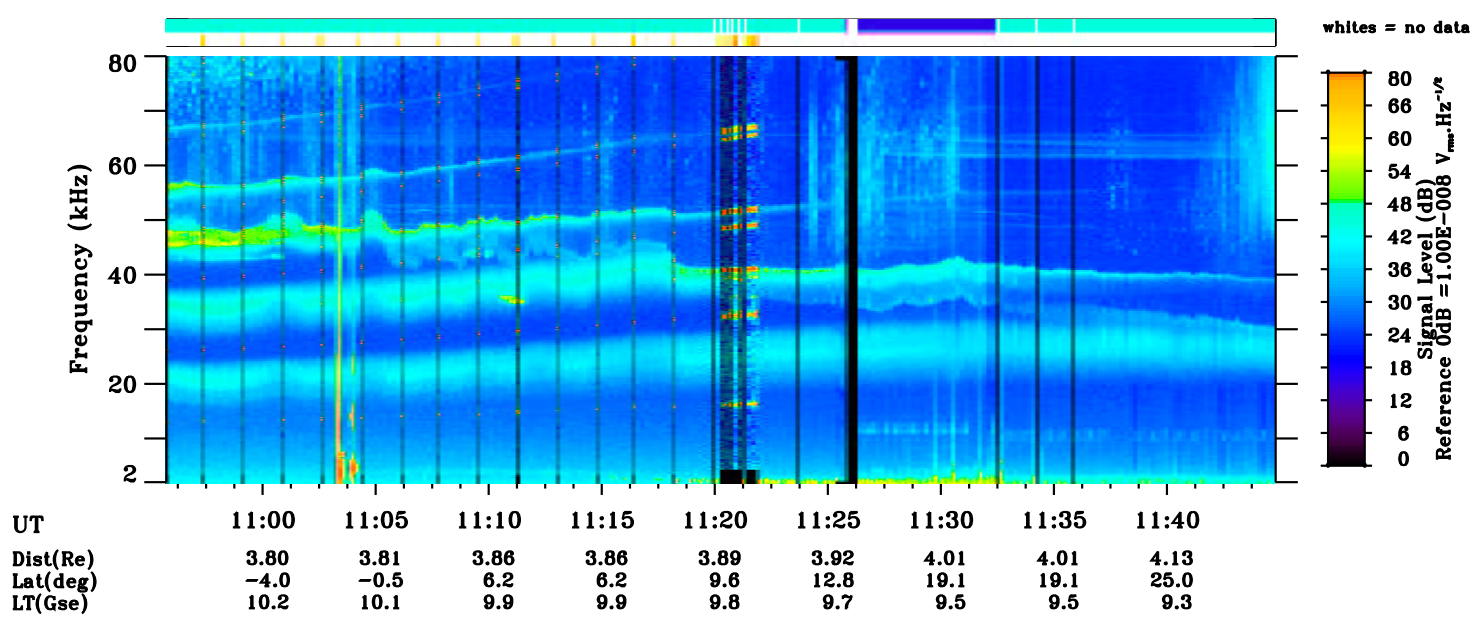

Method: Last

01 OCT 2000

LPCE/CNRS

Fig. 7. Dynamic spectrogram obtained from Whisper data on 1 October 2000 from Cluster 1 Rumba. The frequency bandwidth of the emissions detected between the harmonic of the gyrofrequency varies from broad band to narrow band during this period. They correspond to the Fqs triggered in the active mode by Whisper. The plasma frequency is identified with the low frequency cutoff of the emission, which decreases in frequency from about $45 \mathrm{kHz}$ at 11:00 UT down to $30 \mathrm{kHz}$ at 11:45 UT.

ing the period to $16.4 \mathrm{kHz}$ at 11:20 UT. The natural spectra are primarily composed of banded emissions. Each band of emission is between two consecutive harmonics and its central frequency increases slightly with the increasing gyrofrequency. Their bandwidth tends to decrease with time, and reduce to a narrow band emission before vanishing in the background noise, at about 11:15 UT for the band above $4 F c$, at about 11:25 UT for the band above $3 F c$ and at the end of the display, at 11:45 UT for the constant frequency band close to $40 \mathrm{kHz}$. A low amplitude fuzzy emission is also seen close to $45 \mathrm{kHz}$ at about 11:05 UT, below the main band observed between $3 F c$ and $4 F c$, with a modulated low frequency cutoff. This cutoff reaches the banded emissions extending below from $2 F c$ to $3 F c$ at 11:18 UT, and is seen with a decreasing frequency down to $30 \mathrm{kHz}$ at 11:35 UT. The continuous operation of the emitter on Whisper between 11:20 UT and 11:22 UT, clearly shows that the banded emissions with decreasing frequency bandwidths are $F q s$, and that the upper cutoff of the banded emission, at $40 \mathrm{kHz}$ at 11:20 UT, for example, exactly matches the strong resonance.

Frequency versus amplitude spectra are displayed in Figs. 8 and 9 to give a more detailed view of these natural emissions at two times around 11:04 UT and 11:22 UT. On the active spectrum, collected at 11:04:26 UT, a few seconds before the natural spectrum at the top of Fig. 8, three resonances can be observed between $3 F c(41.50 \mathrm{kHz})$ and $4 F c(55.18 \mathrm{kHz})$. The two first at $45.9,48.01 \mathrm{kHz}$ have a relatively low amplitude, the third one at $49.48 \mathrm{kHz}$ has an amplitude an order of magnitude above. Using the gyrofrequency derived from the harmonics $(13.8 \mathrm{kHz})$, we can note that the frequency separation between the first two corre- sponds to $F p$ and $F u h\left(45.9^{2}+13.8^{2}=47.93^{2}\right)$ when taking into account the frequency resolution of the instrument $(160 \mathrm{~Hz})$. Note that these two resonances have disappeared two seconds later in the following active spectrum. The other non- $n F c$ resonances are $F q s$ observed at $49.48,57.94$ and $70.48 \mathrm{kHz}$. The lower and higher frequency cutoff of the signal $(45.9$ and $50.8 \mathrm{kHz})$ do match the frequencies of $F p$ and $F q$, when taking into account the small frequency fluctuation seen on consecutive spectra, due to the fast evolution of the plasma parameters, as one can ascertain from Fig. 7. The same agreement is found for the data collected at 11:22 UT in Fig. 9, where the two faint resonances at 36.13 and $39.55 \mathrm{kHz}$ correspond to $F p$ and $F u h\left(36.13^{2}+16.44^{2}=39.69^{2}\right)$ with the gyrofrequency $F c=16.44 \mathrm{kHz}$, derived from the $n F c$ $(16.44,32.88,49.48,65.76 \mathrm{kHz})$, while the others $(41.18$, $52.08,67.06,67.38)$ are $F q s$, with the last one split into two peaks. The natural emissions in this band is spreading between $36.1 \mathrm{kHz}$ and $41.8 \mathrm{kHz}$, with the peak frequency $(41.18 \mathrm{kHz})$ matching exactly the $F q$ resonance. The lower cutoff frequency has a fast varying amplitude and shape, as its frequency decreases, as can be seen a few seconds (11:23:33 UT) later. There is also a strong indication that both resonances and plasma emissions are being spin modulated (not shown here). Further studies will determine the impact on the emission levels of parameters, such as the spin angle relative to the magnetic field, or the frequency position relative to the $n F c$. This example confirms that if resonances and natural plasma emissions are indeed observed at $F p$ and $F u h$, then there are spectra where the peak resonance and natural emissions are, in fact, detected at the first $F q$ frequency, as already suggested by Christiansen et al. (1978b) 

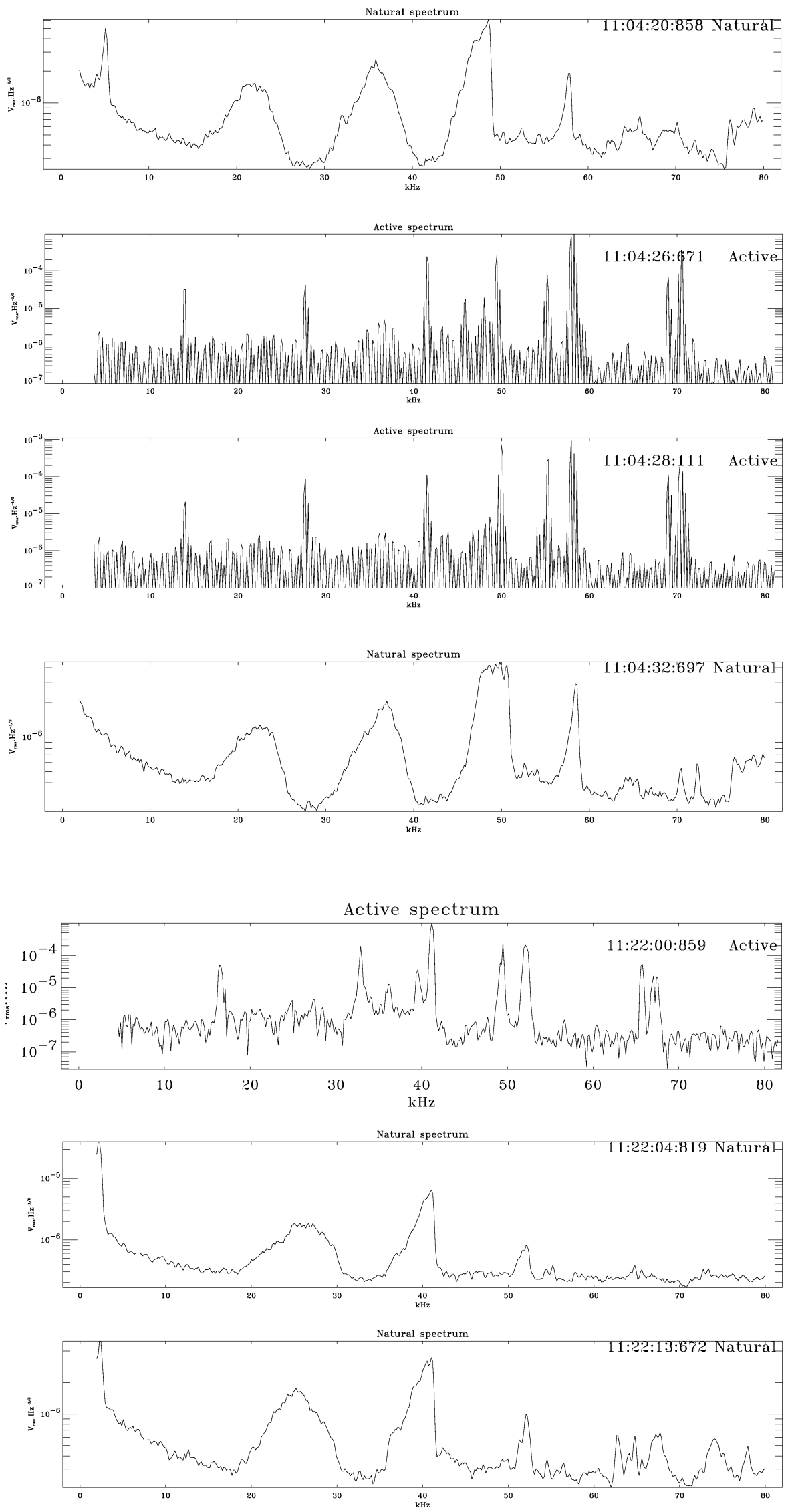

Fig. 8. Consecutive Whisper passive and active spectra from Rumba on 1 October. The natural emissions which lie between $F p$ at $45.9 \mathrm{kHz}$ are seen as a low power resonance on the second active spectrum and with a strong resonance at $F q$ at $49.8 \mathrm{kHz}$. The resonances at $F p$ and $F u h$ have disappeared on the second active spectrum.

Fig. 9. Consecutive active and passive spectra collected at 11:22 UT. Fp and $F u h$ are seen as low amplitude resonances below the strong $F q$. The natural emission lies between $F p$ and $F q$. 

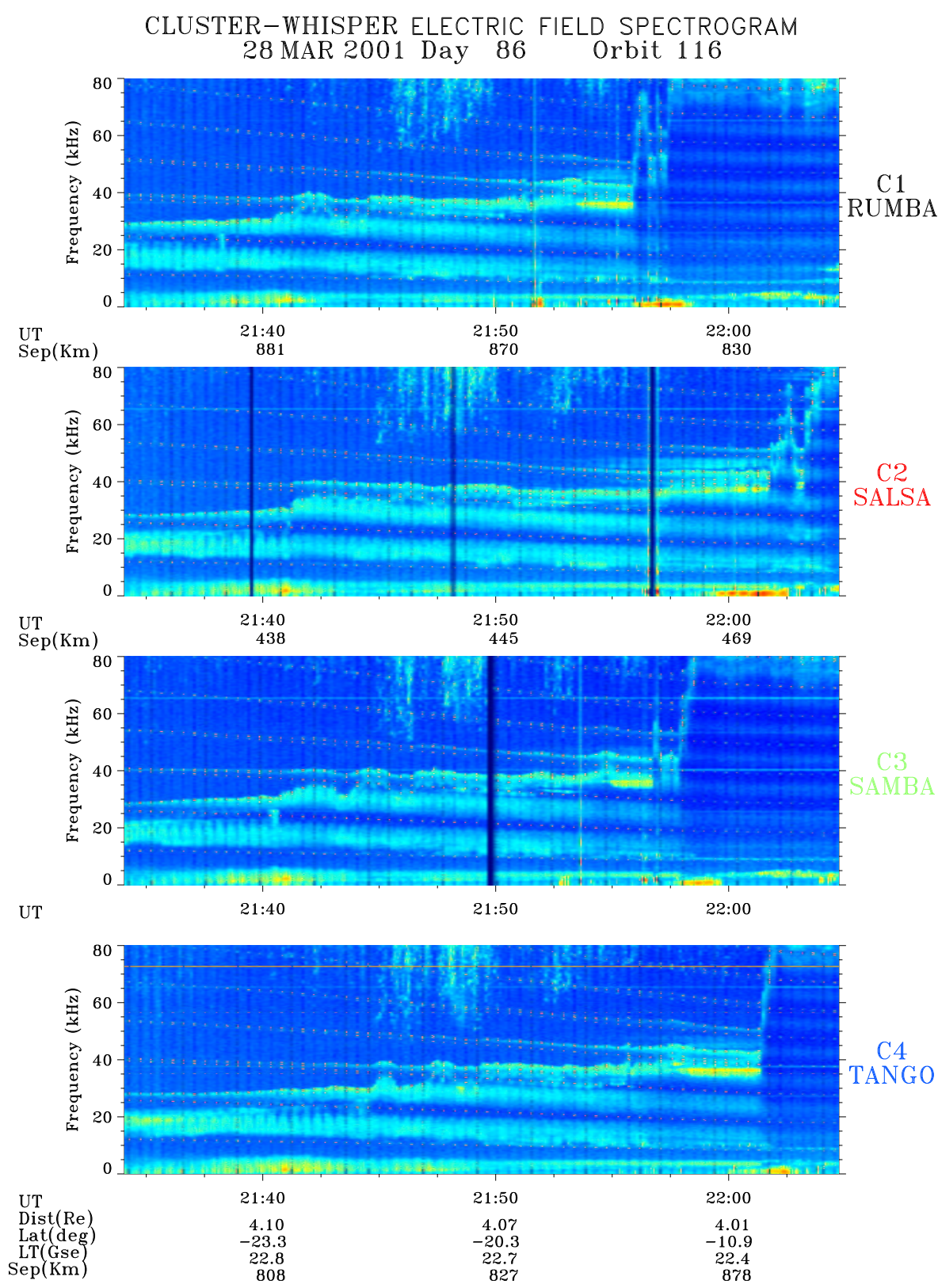

Fig. 10. Dynamic spectrograms obtained from the four spacecraft as they encounter the plasmapause on 28 March. Electric field signal strength ranges from about $5 \cdot 10^{-6}$ (blue) to $5 \cdot 10^{-4} \mathrm{~V} / \mathrm{Hz}-{ }^{1 / 2}$ (red). Broad band spin modulated emissions are observed below $F p$, which itself is a faint, barely visible emission seen rising on Salsa, from about $25 \mathrm{kHz}$ at 21:40 UT to about $35 \mathrm{kHz}$ at $21: 45 \mathrm{UT}$ and $40 \mathrm{kHz}$ at 22:00 UT. Narrow band $F q s$ are seen above $F p$. The small dots are the resonances triggered by the sounder. The horizontal red bar at $72 \mathrm{kHz}$ on Tango is an interference. and Etcheto et al. (1983).

Figure 10 shows the same signatures, but they come this time from the four spacecraft and illustrate the multi-point view provided by Cluster. On that day, 28 March 2001, the plasmapause was encountered in the nighttime sector, at about 22:30 LT. The spacecraft separation was in the range of 400-800 km. On the dynamic spectrogram, the small dots represent the resonances triggered by the sounder, which was active for $3 \mathrm{~s}$, every $28 \mathrm{~s}$ during that period. The plasmapause encounter occurred with a time difference of a few minutes between the four satellites. The differences in the density fluctuations which can be derived from the emission at $F p$ is clearly visible before this line went out of the Whisper frequency range. Differences can also be observed in the wave spectra gathered before 22:00 UT. The faint haze at the plasma frequency is barely visible, because it is highly spin modulated between the first two broadband emission, reaching about $30 \mathrm{kHz}$ at 21:40 UT for Salsa and Samba, and 21:45 UT on Tango. Fluctuations of $F p$ between the second and third broadband emissions, in the range of $35-40 \mathrm{kHz}$ are more evident after 21:40 UT (21:45 UT for Tango) on all spacecraft. Narrow band emissions are also visible at the $F q s$, above $F p$. The strong emission intensifies just before the first sharp density rise, at about 21:55 UT for Rumba and Samba, and at about 22:00 UT for Salsa and Tango. The reader should also note the small density bump observed at 22:02 UT, for example, on Salsa, before the main plasma frequency jump, which is observed with a different density value (larger on Rumba) and duration (shorter on Samba) and is not seen on Tango. The wave signatures are often 
highly spin modulated, a key information which will be used to combine the four points of view in future studies.

\section{Discussion}

We have seen that in its normal mode of operation, Whisper can observe the various and complex natural wave spectra outside the plasmapause with a good time resolution, frequency and sensitivity, from the single emission close to the Fuh to complex patterns that mix both local banded, broad band or narrow band plasma emissions on the Bernstein modes and propagate electromagnetic banded continuum radiation. The simultaneous observations of these features from the Whisper instruments on board each of the Cluster spacecraft confirm that all of these signatures are detected by the four instruments, and this allows one to track their detailed separated evolution both in space and time, as shown in Fig. 10. These spectra and their differences reveal density variations at the scale of the spacecraft separation, a few hundred kilometers for the data used in Fig. 10, and also suggest small differences in the electron distribution functions encountered at the four locations, which could be investigated by further common studies with the Cluster Peace instruments. These frequently observed emissions can be identified with good confidence when using the characteristic frequencies of the resonances triggered by the relaxation sounder. The resonances at $F p$ and $F u h$ can sometimes be simultaneously observed, as reported here for the first time in the high altitude magnetosphere (see Figs. 2 and 5, for example). Their relationship with natural emissions is more reliably established than could be achieved on previous missions. $F p$ does not appear in these data as a narrow band emission, but rather as the lower cutoff of an enhanced background noise, seen as a faint haze below the main peak on the spectrograms. The strongest emission is confirmed to be either $F u h$ or the first $F q$, and its frequency variations can be monitored down to the instrument time resolution, which is typically $2 \mathrm{~s}$ in normal mode, but can reach $0.3 \mathrm{~s}$ in the Cluster burst mode. Fast monitoring $(\sim 0.1 \mathrm{~s})$ has been performed on Geos (Gough et al., 1981b) during nonstandard operation modes for a limited period, but in order to do this, the frequency bandwidth swept by the instrument had to be decreased to a few hundred $\mathrm{Hz}$, thus the information on the spectrum outside the main peak was lost. The time and frequency resolution are, of course, better when the waveform sampled over the whole frequency range can be downloaded, as on previous spacecraft, such as Isee-1 (Kurth et al., 1981) or on the WBD instrument on Cluster (Gurnett et al., 2001, this issue). But such data are usually available only for limited periods of time. In the gyroharmonic band which contains $F u h$, emissions are continuously observed in the events considered in this paper. One observes amplitudes well above the background noise from the electron plasma frequency, which corresponds to a propagation in the direction parallel to the local magnetic field, up to the $F q$ frequency, which corresponds to a propagation perpendicular to the magnetic field. Enhancements of the emissions at $F p$, $F u h$ or $F q$ can be observed by Whisper, depending on the local plasma parameters which can cause instability in either of these solutions of the dispersion relation. The reader can also note that the so-called diffuse resonances, $\mathrm{Dn}$, triggered below $F p$ by the powerful emitters of the ionospheric topside sounders (McAfee, 1974) were predicted to be excited as well by relaxation sounders in the magnetoshere (Osherovich et al., 1993). These resonances are indeed not observed in the Geos and Isee-1 data (Canu, 2001), and are clearly not present in Whisper data (see Fig. 1 for example).

One strong point in the diagnosis of $F p$ based on resonances observed in Bernstein modes should have been its high redundancy, since each set of $F q s$ for a given spectrum should correspond to a single value of $F p / F c$ when the electron distribution function is Maxwellian (Belmont, 1981). However, this is not verified with the spectra presented in this paper. For example, we can derive the electron density from each of the Fqs reported in Fig. 2 of this paper by a method based on the Hamelin's diagrams. The Hamelin's diagrams provide the locus of the $F q s$ from a given value of $F p / F c$ and are discussed in Belmont (1981) (see also Trotignon et al., 2001, this issue). For the $F q$ frequencies at 54.52 and $68.85 \mathrm{kHz}$ of the top spectrum, this gives an $F p$ value of $35.7 \mathrm{kHz}$ and $31.6 \mathrm{kHz}$, respectively, a value which is not unique and is lower than the observed $F p(44.3 \mathrm{kHz})$. This disagreement repeats itself with the resonances displayed in Fig. 8; the derived $F p$ values from the $F q s$ seen at 11:04:26 UT at the frequencies $49.48,57.94,70.48 \mathrm{kHz}$ are about $42.1,34.2,32.0 \mathrm{kHz}$ compared to the observed value of $45.9 \mathrm{kHz}$. For the active spectrum at 11:22 UT in Fig. 9, $F q$ at $41.18,52.08,67.06 \mathrm{kHz}$ provides respective values of $F p$ at $34.0,29.2,26.8 \mathrm{kHz}$. This has already been reported from Geos data (Etcheto et al., 1983) and can be understood when one considers that the standard Bernstein modes used in Hamelin's diagrams are computed for a Maxwellian distribution function of the electrons, which is barely observed in the magnetosphere. In a multi-temperature isotropic electron distribution function, the solutions in each harmonic branch are modified; the most affected one is the one which contains Fuh (Belmont, 1981). The maximum in frequency tends towards the location of the $F q$, depending primarily on the coldest component of the distribution, when the density and temperature ratio between the cold and hot components is not too small (Belmont, 1981). Observations of both resonances and plasma waves which fit in the multi-component Bernstein modes were common in Geos spacecraft data (Etcheto et al., 1983). A further indication of the impact of a nonMaxwellian electron distribution function is provided by the slight modulation in the frequency observed in the banded emissions below $F p$ in Fig. 7. At that time, no fluctuations were observed on the electron gyrofrequency. These modulations are correlated with the small frequency modulation of $F p$. In a Maxwellian plasma, the Bernstein modes below $F p$ are continuously decreasing in frequency and are almost not at all affected by changes in the density. But this is no longer true in a multi-component electron distribution, 
where a secondary maximum appears, depending on the density and temperature ratios of these components, as shown by Belmont (1981). This qualitative interpretation will be checked in future studies by more quantitative computations of the dispersion relations of waves in a multi-component electron distribution. A final question is related to the existence of additional resonances expected in a non-Maxwellian electron distribution. The modified Bernstein modes which results from this distribution function bring additional frequencies with zero-group velocities (Belmont, 1981); this should result in additional resonances triggered by the relaxation sounder. These resonances, termed "hot Fqs", were indeed observed on Geos (Etcheto et al., 1983) and should appear on Whisper. However, although some resonances seem to exhibit multiple peaks, no specific search has been undertaken in this work. The precise identification of these hot $F q s$, which are generally of low amplitude, requires a high frequency resolution, and the Whisper capability here $(160 \mathrm{~Hz})$ is not reaching the best resolution available on Geos $(12 \mathrm{~Hz})$.

\section{Conclusion}

This first analysis of the plasma waves observed by Whisper on Cluster have shown that the good sensitivity, time and frequency resolution of the instrument, combined with the plasma diagnosis capabilities provided by the resonances triggered by the relaxation sounder, are promising significant advances in the identification on a routine basis of natural plasma emissions. Whisper observational capabilities confirm that emissions at $F p, F u h$ and $F q s$ can sometimes be observed at the same time, with varying relative electric field strength. Most of the time, the emission at the plasma frequency is not observed as a strong, narrow band, peak in the wave spectra, but rather as the lower cutoff of an enhanced background noise, which can be tracked by Whisper. The good frequency resolution of the instrument allows are to discriminate between these various emissions with good confidence, with the help of the resonances triggered in the active mode. The good sensitivity and time resolution permits are to track in detail and over long period, their respective frequency and time evolution depending on the variations of the local plasma parameters. Similar features, as well as differences in their evolution in time and frequency, are already easily observed in the data gathered close to the plasmapause by the four spacecraft, which reveals small differences in the density variations as Cluster approachs the boundary. This first study confirms the conclusions reached from the Geos data that the observed emissions are not fully modeled by the Bernstein modes derived from a single electron distribution function. Future detailed identifications of both resonances and plasma waves should then rely on a multi-temperature distribution of electrons. The capability of Whisper to precisely categorize the various characteristics of the observed emissions and their fast evolution with time allows one to now undertake the investigation of the three- dimensional characteristics of the plasma waves which reslts from the combination of data coming from the four Cluster spacecraft.

Acknowledgements. We are very grateful to all the members of the Cluster project who made this mission a success. We are indebted to all the colleagues who contributed in the design, build (and rebuild), tests and data processing of the Whisper instruments throughout the years, and more specially to all the WEC team. We have a specific thought for L. Woolliscroft former PI of the DWP on WEC who is not sharing with us the excitement of this first contact with the Cluster data. We are very grateful to the people of the WEC team and at ESOC who make the long commissioning phase of WEC instruments a success, more specially to P. A. Linquist, WEC chairman, and Silvano Manganelli from ESOC. We thank the French Space Agency CNES for financial and data analysis support. The CETP contribution to Whisper is supported by CNES under contract 7358 .

The Editor in Chief thanks S. Buchert and another referee for their help in evaluating this paper.

\section{References}

Barbosa, D. D.: Low level VLF and LF radio emissions observed at Earth and Jupiter, Rev. Geophys., 20, 316, 1982.

Belmont, G.: Characteristic frequencies of a non Maxwellian plasma. A method for localizing the exact frequencies of magnetospheric intense natural waves near $F p$, Planet. Space Sci. 29, $1251,1981$.

Belmont, G., Canu, P., Etcheto, J., de Feraudy, H., and Higel, B.: Contribution of relaxation sounding experiments to the understanding of magnetospheric processes, ESA SP-217, 701-704, 1984.

Canu, P., Belmont, G., and Fontaine, D.: Role of the equatorial $\mathrm{ECH}$ waves in the generation of diffuse auroras. Contribution of the Geos IMS spacecraft, ESA SP-217, 581-584, 1984.

Canu, P.: Observation of long-duration gyroharmonic resonances: A refutation of the short duration explanation for interpreting the anomalous URAP sounder spectra observed in the Io torus, Radio Sci., 36, 171-181, 2001.

Christiansen, P. J., Gough, M. P., Martelli, G., Bloch, J. J., Cornilleau, N., Etcheto, J., Gendrin, R., Jones, D., Beghin, C., and Décréau, P. M. E.: Geos, Identification of natural magnetospheric emissions, Nature 272, 682, 1978a.

Christiansen, P. J., Gough, M. P., Martelli, G., Bloch, J. J., Cornilleau, N., Etcheto, J., Gendrin, R., Beghin, C., Décréau, P. M. E., and Jones, D.: Geos-1 observations of electrostatic waves and their relationship with plasma parameters, Space. Sci. Rev., 22, 283-400, 1978b.

Crawford, F. W.: Cyclotron harmonic waves in warm plasma, Radio Sci. 69D, 789, 1964.

Décréau, P. M. E., Hamelin, M., Massif, R., de Feraudy, H., Pawela, E., Perraut, S., Pottelette, R., and Bahnsen, A.: Plasma probing by active experiments on the Viking satellite, Ann. Geophysicae Ser. A, 5, 181-186, 1987.

Décréau, P. M. E., Fergeau, P.,Krasnoselsḱikh, V., Lévêque, M., Martin, P., Randriamboarison, O., Sené, F. X., Trotignon, J. G., Canu, P., and Mögensen, P. B.: Whisper. A resonance sounder and wave analyzer: Performance and perspectives for the Cluster mission, Space Sci. Rev., 79, 157-193, 1997.

Décréau, P. M. E., Canu, P., Le Guirriec, E., Krasnoselsḱikh, V., Randriamboarison, O., Rauch, J. L., Trotignon, J. G., and Whis- 
per experimenters: Early results from the Whisper instrument on Cluster: an overview, Ann. Geophysicae, 2001, this issue.

Etcheto, J. and Bloch, J. J.: Plasma density measurements from the GEOS-1 relaxation sounder, Space Sci. Rev., 22, 597-610, 1978.

Etcheto, J., Christiansen, P. J., Gough, M. P., and Trotignon, J. G.: Terrestrial continuum radiation observations with Geos-1 and Isee-1, Geophys. Res. Lett., 9, 1239-1242, 1982.

Etcheto, J., Belmont, G., Canu, P., and Trotignon, J. G.: Active sounder experiments on GEOS and ISEE, ESA SP-195, 39-46, 1983.

Etcheto J., Christiansen, P. J., and Gough, M. P.: Problems in the interpretation of natural plasma wave spectra, Ann. Geophysicae, 3, 4, 417-420, 1985.

Gough, M. P., Christiansen, P. J., Martelli, G., and Gershuny, E. J.: Interaction of electrostatic waves with warm electrons at the geomagnetic equator, Nature, 279, 515, 1979.

Gough, M. P., Christiansen, P. J., and Gershuny, E.: Electrostatic wave morphology near the geostationnary orbit, Adv. Space Res., 1, 337, 1981a.

Gough, M. P., Christiansen, P. J., and Thomas, R.: Electrostatic emissions studied in high rsolution, Adv. Space. Res., 1, 345, 1981b.

Gurnett, D. A.: The Earth as a radio source: The non thermal continuum, J. Geophys. Res., 80, 2751-2763, 1975.

Gurnett D. A. , Huff R. L., Pickett, J. S., Persoon, A. M., Kletzing, C. A., Mutel, R. L., Christopher, I. W., Inan, U. S., Martin, W. L., and Bougeret, J.-L.: First Results from the Cluster Wide-Band Plasma Wave Investigation, Ann. Geophysicae, 2001, this issue.

Gustafsson, G., Boström, R., Holback, B., Holmgren, G., Lundgren, A., Stasiewicz, K., Ahlen, L., Mozer, F. S., Pankow, D., Harvey, P., Berg, P., Ulrich, R., Pedersen, A., Schmidt, R., Butler, A., Fransen, A. W. C., Klinge, D., Thomsen, M., Fâlthammar, C. G., Lindqvist, P.-A., Christenson, S., Holtet, J., Lybekk, B., Sten, T. A., Tanskanen, P., Lappalainen, K., and Wygant, J.: The electric filed and wave experiment for the Cluster mission, Space Science Reviews, 79, 137-156, 1997.

Gustafsson, G., André, M., Eriksson, A. I., et al.: First results of electric field and density oberservations by Cluster EFW based on initial months of operation, Ann. Geophysicae, this issue, 2001.

Higel, B.: Small scale structure of magnetospheric electron density through on-line tracking of plasma resonance, Space Sci. Rev. 22, 611-631, 1978.

Jones, D.: Terrestrial myriametric radiation from the Earth's plasmapause, Planet. Space Sci., 30, 339, 1982.

Kurth, W. S.: Detailled observations of the source of terrestrial narrowband electromagnetic radiation, Geophys. Res. Lett., 9, 1341-1344, 1981.

LaBelle, J., Ruppert, D. R., and Treuman, R. A.: A statisitical study of banded magnetospheric emissions, J. Geophy. Res., 104, 293 303, 1999.

Le Sager, P., Canu, P., and Cornilleau-Wehrlin, N.: Impact of the Ulysses velocity on the diagnosis of the electron density by the Unfied Radio and Plasma Wave sounder in the outskirts of the Io torus, J. Geophys. Res., 103, 26 667, 1998.

McAfee, J. R.: Electron plasma resonances in the topside ionosphere, Fundam. Cosmic Phys., 1, 71-117, 1974.

Meyer-Vernet, N., Hoang, S., and Moncuquet, M.: Bernstein waves in the Io plasma torus: A novel kind of electron temperature sensor, J. Geophys. Res., 98, 21 163-21 176, 1993.

Moncuquet, M., Meyer-Vernet, N., Hoang, S., Forsyth, R. J., and Canu, P.: Detection of Bernstein wave forbidden bands in the Jovian magnetosphere: A new way to measure the electron density, J. Geophys. Res., 102, 2373-2379, 1997.

Osherovich, V. A., Benson, R. F., Fainberg, J., Stone, R. G., and MacDowall, R. J.: Sounder stimulated Dn Resonances in Jupiterś Io torus, J. Geophys. Res., 98, 18 751-18 756, 1993.

Pedersen, A., Cornilleau-Wehrlin, N., de La Porte, B., Roux, A., Bouabdellah, A., Décréau, P. M. E., Lefeuvre, F., Sené, F. X., Gurnett, D., Huff, R., Gustafsson, G., Holmgren, G., Woolsiscroft, L., Alleyne, H. St. C., Thomson, J. A., and Davies, P. H. N.: The Wave Experiment Consortium (WEC), Space Sci. Rev., 97, 93-106, 1997.

Perraut, S., De Feraudy, H., Roux, A., Décréau, P. M. E., Paris, J., and Matson, L.: Density measurements in key regions of the Earth's magnetosphere: Cusp and auroral region, J. Geophys. Res., 95, 5997-6014, 1990.

Trotignon, J. G., Décréau, P. M. E., Rauch, J. L., Randriamboarison, O., Krasnoselskikh, V., Canu, P., Alleyne, H., Yearby, K., Le Guirriec, E., Séran, H. C.,Sené, F. X., Martin, Ph., Lévêque, M., and Fergeau, P.: How to determine the thermal electron density and the magnetic field strength from the Cluster/WHISPER observations around the Earth, Ann. Geophysicae, this issue, 2001.

Woolliscroft, L. J. C., Alleyne, H. St. C., Dunford, C. M., Sumner, A., Thomson, J. A., Walker, S. N., Yearby, K. H., Buckley, A., Chapman, S., Gough, M. P., and the DWP co-investigators: The Digital Wave-Processing Experiment on Cluster, Space Sci. Rev., 97, 209-231, 1997. 